\title{
POSIÇÃO CORPORAL E OXIGENAÇÃO PARTE I - PACIENTES COM DISFUNÇÃO PULMONAR UNILATERAL
}

\author{
Cibele Andrucioli de Mattos Pimenta* \\ Maria Sumie Koizumi**
}

PIMENTA, C.A. de M. \& KOIZUMI, M.S. Posição corporal e oxigenação: pacientes com disfunção pulmonar unilateral - parte 1. Rev. Esc. Enf. USP, São Paulo, 23(3):205-221, dez. 1989.

Este estudo verificou o efeito das posiçōes sentada, supina e laterais sobre os gases do sangue arterial, em pacientes com disfunçāo pulmonar unilateral, no pós-operatório recente de cirurgia cardlaca. Os gases sangülneos foram mensurados após o paciente permanecer 15 minutos em cada posição. Concluiu-se que quando o paciente deitava-se sobre o lado do pulmão considerado sadio ou em supino, a Pa02 foi significativamente superior. No entanto, na posição deitada sobre o lado afetado, houve piora significativa da PaO2. de posição.

UNITERMOS: Gasometria - sangue. Posiçâo corporal. Paciente, mudançat

\section{INTRODUÇÃO}

$\mathrm{O}$ atendimento de pacientes com alteraçōes da função pulmonar, como doença de base ou como complicação desenvolvida durante o curso de outra moléstia, é atividade cotidiana do enfermeiro.

Em pacientes que se submeteram à cirurgia, fatores relativos ao próprio ato anestésico, a imobilidade e a dor, entre outros, os predispõem a apresentarem complicações pulmonares.

Dentre os procedimentos utilizados para a assistência pulmonar a esses pacientes, a mudança de decúbito a intervalos pré-determinados, é conduta largamente difundida na prática da enfermagem, como uma rotina. À enfermeira compete determinar as posiçōes a serem adotadas e a freqüência de sua mudança.

Em pacientes acamados, a mudança de decúbito interfere na distribuição da ventilação e perfusão pulmonares, resultando numa diminuição do "shunt" arteriovenoso e melhora dos níveis de oxigenaçāo sangüínea (RAY et alii, 1974).

\footnotetext{
* Professor Assistente da Escola de Enfermagem da USP. Departamento de Enfermagem Medico-Cirúrgica.

** Professor Assistente Doutor da Escola de Enfermagem da USP. Departamento de Enfermagem MédicoCirúrgica.
}

Rev. Esc. Enf. USP, São Paulo, 23(3):205-221, dez. 1989 
Em indivíduos adultos sadios, a posição do corpo não altera a oxigenação, segundo os achados de ZACK et alii (1974) e REMOLINA et alii (1984), fato confirmado por KAHN et alii (1985) e PEIRANO et alii (1986).

No entanto, em indivíduos com alteraçōes pulmonares, estudos têm demonstrado que posicionar o paciente levando em conta a área pulmonar afetada pode prevenir a hipoxemia e melhorar a oxigenação sangüínea (ZACK et alii 1974; KATZ \& BARASH 1977; SEATON et alii 1979; DHAINAUT et alii 1980; IBANEZ et alii 1981; REMOLINA et alii 1984; BIDIWALA et alii 1984; NEAGLEY \& ZWILLICH 1985).

Apesar dos trabalhos existentes, na assistência de enfermagem a pacientes com problemas pulmonares, pouco se comenta sobre o uso deliberado da posição corporal como forma de otimizar a relação ventilação e perfusão e portanto, a troca gasosa (DEAN 1985; GROSMAIRE 1983).

NG \& McCORMICK (1982) em seu artigo sobre as alterações fisiológicas devidas à mudança de posição, apontam que a distribuição da ventilação depende da complacência pulmonar, da resistência das vias aéreas e da posição do corpo. A distribuição da perfusão é afetada pelo débito cardíaco, pela resistência vascular pulmonar e posição do corpo.

A distribuição da ventilação de acordo com a posição do corpo é a seguinte:

- na posição ereta, em condições normais, esta diminui da base para o ápice, embora as diferenças sejam menores que as da perfusão (KANEKO et alii 1966; WEST 1977b; WEST \& DOLLERY 1960). A pressão intrapleural é maior na base $(-2,5 \mathrm{cmH} 2 \mathrm{O})$ que no ápice $(-10 \mathrm{cmH} 2 \mathrm{O})$ do pulmão. Isto é, na base a pressāo intrapleural é menos sub-atmosférica que no ápice. Possivelmente, o peso do pulmão sobre si mesmo, é o responsável por esta diferença. Devido a isto, os alvéolos da base expandem-se melhor que os do ápice (WAGNER \& WEST 1979);

- nas posiçōes supina e prona, em condiçōes normais, a diferença da pressão intrapleural entre o ápice e a base diminui, e a distribuição torna-se mais uniforme, mas ainda assim, permanece melhor na base. No entanto, a ventilação da porção posterior excede à da anterior (KANEKO et alii 1966; WAGNER \& WEST 1979).

- na posição lateral, em condições normais, o pulmão que fica em plano inferior é melhor ventilado que o superior. $O$ hemidiafragma da região inferior é empurrado para dentro do tórax, pelas vísceras abdominais. Esta compressão levao a se contrair com maior efetividade (WAGNER \& WEST 1979; KATZ \& BARASH 1977).

Além disso, na respiração espontânea, em indivíduos acordados, há melhor contração do diafragma das regiōes pulmonares que estiverem em plano inferior, levando a uma maior ventilação destas áreas. Em situação de ventilação mecânica ou durante a anestesia, este fato não ocorre (FROESE \& BRYAN, 1974). 
A distribuição da perfusão, de acordo com a posição do corpo é a seguinte:

- na posição ereta, em condiçōes normais, o fluxo sangüíneo diminui da base para o ápice do pulmão (WEST 1977b; WEST \& DOLLERY 1960);

- na posição supina, o fluxo na região apical e na base pulmonar são semelhantes; entretanto, o fluxo na região posterior excede o da anterior (WEST 1977b; WEST \& DOLLERY 1960);

- nas posiçōes lateral e prona, a região inferior do pulmão é melhor perfundida que a regiâo superior (KANEKO et alii 1966).

Tais diferenças são explicadas pelo efeito da gravidade sobre a pressão hidrostática nos vasos sangüíneos pulmonares. $\mathrm{Na}$ posição ereta, a pressão no sistema arterial diminui aproximadamente $1 \mathrm{cmH} 20$ por $\mathrm{cm}$ de distância, na direção da base para o ápice. Isto explica a maior perfusão da base pulmonar. Assim, teoricamente, chega-se a um ponto em que a pressão alveolar excede à capilar, e estes tendem a colabar. No entanto, em condiçōes fisiológicas, esta situação de regiōes não perfundidas, nāo ocorre (WEST 1977b).

Os dados mencionados fundamentam o uso deliberado da posição corpórea, como forma de interferir nos níveis de oxigenação sangüínea. A partir deles, desenvolveu-se este estudo.

Diante dos estudos realizados percebe-se que o uso da posição como recurso terapêutico para melhorar a oxigenação sangüínea ou prevenir hipóxia, pode ser um dado de grande valor no cuidado de pacientes com problemas pulmonares, embora muitos aspectos precisem ainda de melhores esclarecimentos. Sobretudo, chama a atenção, o fato de não se ter encontrado trabalho nacional sobre o assunto.

$\mathrm{O}$ efeito da posição semi-sentada sobre a $\mathrm{PaO} 2$ em pacientes com doença pulmonar unilateral permanece ainda obscuro. No entanto, esta posição é muitas vezes a de primeira escolha dos enfermeiros, no cuidado de seus pacientes.

Outro fato também verificado nos estudos realizados, foi a falta de homogeneidade da populaçāo NORTON \& CONFORTI (1985) confirmam que há dificuldade de se obter população homogênea em estudos clínicos, principalmente em pacientes críticos; visto a variedade e complexidade de agravos e tratamentos a que estes são submetidos. Comentam que muitos trabalhos foram realizados com população pequena e não homogênea, e recomendam que sejam feitos estudos que testem grupos com problemas similares, tais como: dor, intervenção cirúrgica, hipoventilação, ventilação mecânica, PEEP e gravidade da doença.

Frente às lacunas apontadas, e por se observar que pacientes em pós operatório recente de cirurgia cardíaca apresentam com freqüência disfunçōes pulmonares, julgou-se importante estudar nestes pacientes o efeito das posiçōes corpóreas sobre os gases sangüíneos. Além disso, a insuficiência de informação na literatura 
sobre o uso de decúbitos como um agente terapêutico, dificulta o planejamento do cuidado a tais pacientes, assim como o ensino deste recurso.

O estudo abrangeu as posiçōes sentada, supina e laterais pois, além de apresentarem aspectos que ainda necessitam esclarecimentos, são as tradicionalmente utilizadas no cuidado dos pacientes acamados. Assim, um dos objetivos deste estudo foi verificar o efeito das posiçōes supina, sentada e laterais, sobre a $\mathrm{PaO} 2$ e $\mathrm{PaCO} 2$, em pacientes com disfunção pulmonar exclusiva ou predominantemente unilateral.

Embora o estudo tenha também abrangido portadores de disfunção pulmonar bilateral, os resultados obtidos serão apresentados em um artigo posterior.

\section{METODOLOGIA}

\section{POPULAÇÃO}

Os dados deste estudo foram colhidos na Unidade de Recuperação de Cirurgia Cardíaca do Instituto do Coração da Faculdade de Medicina da Universidade de São Paulo, no período de janeiro a março de 1987.

Para a realização do estudo foi obtida a autorização dos responsáveis clínico e administrativo da unidade e aos pacientes conscientes foi solicitada a sua participação.

A populaçāo foi composta por 21 pacientes internados naquela unidade durante o período do estudo, e os critérios de inclusão utilizados foram: ser adulto, de ambos os sexos, estar em período pós-operatório recente de cirurgia cardíaca e apresentar alguma disfunção pulmonar unilateral.

O Dicionário Médico BLAKESTON (1982), define disfunção como "qualquer anomalia ou comprometimento de uma função ou de um órgão". Neste trabalho foi adotada a mesma definição.

O diagnóstico da disfunção pulmonar foi feito por um médico da unidade, através da avaliação da radiografia de tórax realizada no dia. Considerou-se disfunção pulmonar unilateral qualquer alteração radiológica presente exclusiva ou predominantemente em um dos pulmōes.

\section{MÉTODOS}

Os dados foram colhidos pela autora, e registrados em ficha própria, constituída de 4 partes (Anexo I): dados de identificaçāo, dados de caracterização da população; dados relativos à posição do paciente e respectiva mensuração da $\mathrm{PaO} 2$ e $\mathrm{PaCO} 2$ pelo método de gasometria e registro de intercorrências. 


\subsubsection{Material Utilizado}

- Seringas de $3 \mathrm{ml}$ descartáveis

- Seringas de $10 \mathrm{ml}$ descartáveis

- Agulhas 30x7 descartáveis

- Dispositivo para infusão venosa em asa de borboleta no 21 ou 23

- Rolhas de cortiça

- Heparina $(5.000 \mathrm{U} / \mathrm{ml})$

- Algodão embebido em álcool iodado

- Coxim cilíndrico de $15 \times 50 \mathrm{~cm}$

- Travesseiro de $29 \times 19 \times 8 \mathrm{~cm}$

\subsubsection{Procedimentos Utilizados na Coleta de Dados}

Os pacientes foram posicionados seqüencialmente nos seguintes decúbitos: sentado, supino ou dorsal, lateral direito e lateral esquerdo, e permaneciam por 15 minutos em cada posição. Foi estabelecido este período de tempo por se ter encontrado na literatura, diversos autores que o utilizaram (ZACK et alii 1974; SEATON et alii 1979; IBANEZ et alii 1981) e outros que usaram períodos próximos a este, ou seja, 10 minutos (MARTI \& ULMER 1982 e REMOLINA et alii 1984).

Além disso, NEAGLEY \& ZWILLICH (1985) mediram a saturação de oxigênio 5,10 e 15 minutos após o paciente assumir cada posição, e não encontraram diferenças significativas nos valores da $\mathrm{SaO} 2$, nos três períodos.

Caso o paciente já estivesse na posição sentada, iniciou-se o procedimento pela posição seguinte, ou seja supina.

Após 15 minutos em cada posição, foi feita a coleta de sangue arterial através de punção ou aspiração por cateter arterial. Durante o período em que o paciente permaneceu na mesma posição, não foi submetido à tapotagem, aspiração oro-traqueal, mudança da oxigenoterapia ou qualquer outra manobra que pudesse provocar hipóxia ou hiperóxia e portanto, interferisse no efeito do decúbito sobre a $\mathrm{PaO} 2$ ou $\mathrm{PaCO} 2$.

Nos casos em que houve necessidade de se fazer aspiração ou tapotagem, esperou-se 30 minutos, visando minimizar a hipóxia provocada por estes procedimentos, (GOODNOUGH 1985) e após, efetuou-se a coleta das amostras.

Nos casos de qualquer alteração da oxigenoterapia ou transfusão de sangue, a coleta de dados foi reiniciada, ou o paciente foi excluído do estudo. 
Durante o estudo, os pacientes receberam todas as drogas previstas no seu plano terapêutico, tais como: cardiotônicos, antiarrítmicos, broncodilatadores, sedativos, drogas vasoativas, diuréticos, dentre outras.

Assim, foram colhidas 4 amostras de sangue arterial de cada paciente, correspondendo a cada uma das posiçōes corpóreas selecionadas: sentada, supina e laterais. Cada paciente foi seu próprio controle em relação aos resultados da $\mathrm{PaO} 2$ e da $\mathrm{PaCO} 2$ obtidos em cada posição.

\subsubsection{Análise Gasométrica}

A análise dos gases foi realizada em aparelho de gasometria marca Radiometer, modelo $\mathrm{ABL} 300$, computadorizado, e com eletrodos específicos para mensurações de $\mathrm{pH}, \mathrm{pCO} 2$ e pO2. As seringas utilizadas para a coleta do sangue arterial foram previamente heparinizadas com $0,3 \mathrm{ml}$.

\subsubsection{Tratamento dos dados}

Os dados foram analisados da seguinte maneira:

1 - Dados referentes à caracterização da população (idade, sexo, disfunção pulmonar, drenos toráxicos, tipo de oxigenoterapia), baseando-se em números absolutos e percentuais;

2 - Dados relativos à posição dos pacientes e respectivas $\mathrm{PaO} 2$ e $\mathrm{PaCO} 2$, através de uma análise descritiva de modo exploratório, com a determinação de: Mediana, Média, Desvio padrão e Soma dos postos.

Para a análise e tratamento dos dados utilizou-se nas posições laterais o referencial deitado sobre o lado afetado e deitado sobre o lado não afetado.

$\mathrm{Na}$ análise estatística utilizou-se o Teste de Friedman, não paramétrico, pois a população apresentou uma distribuição com pequena fuga da normalidade e alguns pontos discrepantes, que persistiram, mesmo após transformações. Quando o Teste de Friedman apreendeu diferenças entre as posições, utilizou-se o Teste de Comparaçōes Múltiplas, dois a dois, para detectar onde ocorreram as diferenças, baseando-se em CONOVER (1980).

- Soma dos Postos

$\mathrm{Na}$ Soma dos Postos, foram atribuídos postos de 1 a 4 à $\mathrm{PaO} 2$ e $\mathrm{PaCO} 2$, em cada posição e para cada paciente, nos dois grupos. Assim, para a $\mathrm{PaO} 2$, um determinado paciente recebia o posto mais baixo (1), para a posição que apresentasse o mais baixo nível de oxigênio; o segundo posto mais baixo (2), para o segundo nível mais baixo de oxigênio e assim sucessivamente, até receber o posto mais alto (4), para o nível mais alto de oxigênio. Após, procedia-se à soma dos postos da seguinte maneira: fixava-se uma determinada posição e somavam-se os postos a ela atribuídos, em um determinado grupo. Para a $\mathrm{PaCO} 2$, o procedimento foi o mesmo, apenas invertendo-se a ordem de atribuição dos postos. Se duas posições empatavam na atribuição dos postos, utilizou-se a média dos postos que lhes caberiam $(2,5 ; 3,5$ etc. $)$. 


\section{RESULTADOS E DISCUSSĀO}

Os resultados obtidos estão apresentados em forma de tabelas e quadros e a discussão dos dados é feita concomitantemente.

TABELA 1

DISTRIBUIÇĀO DOS PACIENTES SEGUNDO IDADE E SEXO

SÃO PAULO, 1987

IDADE

MASC.

FEM.

TOTAL

$\begin{array}{rrlllrl} & \text { no } & \% & \text { no } & \% & \text { no } & \% \\ 17-44 & 4 & 19,0 & 4 & 19,0 & 8 & 38,0 \\ 45-67 & 10 & 47,7 & 3 & 14,3 & 13 & 62,0\end{array}$

TOTAL

14

66,7

7

33,3

21

100,0

Os dados da Tabela 1 mostram predomínio na faixa etária de 45 a 67 anos $(62,0 \%)$ e do sexo masculino $(66,7 \%)$.

TABELA 2

ALTERAÇÕES NA IMAGEM RADIOLÓGICA DOS PACIENTES SÃO PAULO, 1987

IMAGEM RADIOLÓGICA

no

$\%$

Atelectasia

10

34,5

Congestão pulmonar

10

34,5

Derrame pleural

9

31,0

TOTAL

29

100,0

A Tabela 2 mostra que as alterações radiológicas mais freqüentes foram atelectasia e congestão pulmonar $(34,5 \%)$. 
Cumpre mencionar que todas as radiografias foram tiradas com o paciente no leito, o que pode comprometer a sua qualidade. Acrescido à isto, o fato da radiografia ter sido feita somente na posição antero posterior; dificultou a caracterização exata da área pulmonar. No entanto, todos os pacientes apresentaram algum grau de comprometimento da base do pulmão considerado afetado.

Dentre os autores que estudaram os efeitos da posição sobre $\mathrm{PaO} 2$ em pacientes com doença pulmonar unilateral temos: SEATON et alii (1979) que trabalharam com portadores de tumor pulmonar submetidos à toracotomia; DHAINAUT et alii (1980) que pesquisaram pacientes com pneumonia; ZACK et alii (1974); IBANEZ et alii (1981) e REMOLINA et alii (1984) que trabalharam com pacientes portadores de diversos diagnósticos: câncer, penumonia, fibrose e embolia pulmonar, broncopneumonia, contusão, hemorragia, enfisema e abcesso pulmonar.

Percebemos que quanto ao diagnóstico pulmonar, a população de nosso estudo difere das demais, que por sua vez, diferem entre si.

É importante lembrarmos que a nossa população, possuía como doenças de base alteraçōes cardíacas e não pulmonares. Provavelmente por isso, as alterações radiográficas encontradas, são menos específicas e possivelmente menos graves, que as de outros estudos.

\author{
TABELA 3 \\ DISTRIBUIÇĀO DOS PACIENTES SEGUNDO A \\ LOCALIZAÇÃO DOS DRENOS TORÁCICOS \\ SÃO PAULO, 1987
}

DRENOS

no

$\%$

Mediano + pleural

12

57,2

Mediano

8

38,0

Ausente

1

4,8

TOTAL

Nota-se pela Tabela 3 que a maioria dos pacientes $(95,2 \%)$, possuía um dreno torácico de localização mediana. Destes, $57,2 \%$ possuíam 2 drenos (um com localização mediana e outro pleural) e somente 1 paciente $(4,8 \%)$, estava sem dreno. 
Esta alta ocorrência de drenos está relacionada com a característica de população do estudo, visto que $20(95,2 \%)$ dos 21 pacientes, estavam nas primeiras 48 horas do período pós-operatório e neste período, é bastante comum a presença dos drenos colocados durante $o$ ato cirúrgico. $O$ paciente que não possuía dreno, estava no 60 dia de pós-operatório.

$\mathrm{Na}$ revisão de literatura não se encontrou referência à presença de drenos torácicos nos pacientes estudados.

No entanto, acredita-se que este dado é importante visto que a presença de dreno, pela dor que ocasiona e pela possível alteração da pressão intrapleural que acarreta, entre outros, talvez interfira na dinâmica respiratória. A maioria dos pacientes (81\%), estavam despertos e em condiçōes de expressar queixas relativas à dor, desconforto intenso e outras; no entanto, queixas específicas não ocorreram, embora alguns pacientes permanecessem gementes todo o tempo.

Não nos foi possível cruzar os dados se os pacientes com diferentes tipos de drenos ou sem dreno, apresentaram respostas diferentes à posição, devido ao pequeno número de ocorrências em algumas situações.

\author{
TABELA 4 \\ DISTRIBUIÇÃO DOS PACIENTES SEGUNDO O \\ TIPO DE OXIGENOTERAPIA RECEBIDA \\ SÃO PAULO, 1987
}

OXIGENOTERAPIA no $\quad$ no

Nebulização

$16 \quad 76,2$

Ventilador mecânico

2

9,5

Nenhum

3

14,3

TOTAL

Observando a Tabela 4 , notamos que $85,7 \%$ dos pacientes recebiam oxigenoterapia. Quanto ao tipo de terapia por oxigênio a que os pacientes eram submetidos, verificamos que houve predomínio de nebulização $(76,2 \%)$ quer seja por máscara ou nebulização à distância. A seguir temos a ocorrência de ventilador artificial $(9,5 \%)$. Encontramos 3 pacientes $(14,3 \%)$ que não recebiam oxigenoterapia, ou seja, estavam respirando espontaneamente em ar ambiente. 
Cabe ressaltar que embora a nebulização por máscara e à distância fossem feitas com $\mathrm{O}_{2}$ a $100 \%$, a fração inspirado final de $\mathrm{O}_{2}$, deve ter variado grandemente devido à fatores como: fluxo de nebulização, adaptação da máscara, deslocamento de correntes de ar, entre outros.

A nebulização à distância era feita, aproximadamente, de 10 a $15 \mathrm{~cm}$ do nariz.

Pelo tipo de oxigenoterapia recebida, acrescido à pouca especificidade das alteraçōes pulmonares encontradas, pode-se pensar que a população deste estudo, em sua grande maioria, era portadora de uma disfunção pulmonar de leve a moderada.

Em relação ao efeito das posições corporais sobre $\mathrm{PaO} 2$ e $\mathrm{PaCO} 2$, nos pacientes com disfunção pulmonar unilateral, pode-se observar seu comportamento através de diversos indicadores nos Quadros 1,2 e 3.

\section{QUADRO 1 \\ COMPORTAMENTO DE PaO2 EM RELAÇÃO ÀS POSIÇÕES SÃO PAULO, 1987}

\begin{tabular}{|c|c|c|c|c|}
\hline & Sentada & Supina & $\begin{array}{l}\text { Lado não } \\
\text { afetado }\end{array}$ & Lado afetado \\
\hline Mediana & 75,50 & 89,50 & 86,00 & 77,00 \\
\hline Média & 91,27 & 92,91 & 93,48 & 86,96 \\
\hline Desvio padrão & 36,03 & 30,23 & 34,87 & 27,10 \\
\hline $\begin{array}{l}\text { Soma dos } \\
\text { postos } \\
\text { № de vezes }\end{array}$ & 52,50 & 59,00 & 59,50 & 39,00 \\
\hline $\begin{array}{l}2 \mathrm{MP}^{*} \\
\text { № de vezes }\end{array}$ & 9 & 14 & 13 & 6 \\
\hline $\mathrm{PP} * *$ & 4 & 4 & 2 & 11 \\
\hline
\end{tabular}

* MP - melhores posiçōes

** PP - pior posição
$\mathrm{F}=2,760$
0,$05 ; 3 ; 60$

Pelo teste de Friedman conclui-se que não há igualdade entre as posiçōes, ao nível de significância de $5 \%$. 
Dada a não igualdade, para detectarmos onde ocorre a diferença, aplicou-se o método de comparação múltipla, dois a dois, no resultado da soma dos postos.

\section{QUADRO 2 \\ COMPARAÇĀO DA SOMA DOS POSTOS NAS QUATRO POSIÇŌES SÃO PAULO, 1987}

Sentada Supina $\quad \begin{aligned} & \text { Lado não } \\ & \text { afetado }\end{aligned}$

\begin{tabular}{lrrrr} 
Sentada & - & 6,5 & 7,0 & 13,5 \\
$\begin{array}{l}\text { Supina } \\
\text { Lado não }\end{array}$ & 6,5 & - & 0,5 & 20,0 \\
afetado & 7,0 & 0,5 & - & 20,5 \\
Lado afetado & 13,5 & 20,0 & 20,5 & - \\
\hline
\end{tabular}

$\mathrm{t}=2,000$

0,$975 ; 60$

$\mathrm{t}=2,390$

0,$98 ; 60$ corresponde $K=15,960$

0,975

corresponde $\mathrm{K}=19,073$

0,98

Pelo Quadro 2 constata-se que as posições deitado sobre o lado não afetado e supina, não significantemente superiores à posição deitada sobre o lado afetado, ao nível de significância de $5 \%$ e mesmo de $2 \%$. As demais comparações não resultaram em diferenças significantes.

Esses resultados estão de acordo com os encontrados por diversos autores.

Assim, em relaçāo à posição deitado sobre o lado não afetado, ZACK et alii (1974); KATZ \& BARASH (1977); DHAINAUT et alii (1980); IBANEZ et alii (1981) e REMOLINA et alii (1984), também encontraram aumento da $\mathrm{PaO} 2$, estatisticamente significativo, quando os pacientes estavam nessa posição.

Neste estudo, três pacientes possuiam dreno pleural no pulmāo considerado sadio, e mesmo assim, apresentaram melhora da $\mathrm{PaO} 2$ ao deitarem-se sobre o lado não afetado.

Em relação à posição deitado sobre o lado afetado, os mesmos autores acima citados, acrescido de NEAGLEY \& ZWILLICH (1985), também encontraram queda na $\mathrm{PaO} 2$, estatisticamente significativa. 
Somente SEATON et alii (1979) não encontraram diferenças na $\mathrm{PaO} 2$ quando compararam as posições deitado sobre o lado não afetado e deitado sobre o lado afetado.

Provavelmente os mecanismos responsáveis pela mudança na $\mathrm{PaO} 2$, quando o paciente deita-se na posição lateral são as modificações na perfusão e ventilação. Assim, quando o pulmão afetado está embaixo, pela ação da gravidade, há aumento do fluxo de sangue para esta área. Embora fatores como a hipoxia e acidose possam afetar a distribuição d perfusāo, ARBORELIUS et alii (1960), demonstraram que estes não superam o efeito da gravidade. No entanto, a ventilação, que em condiçōes normais, é maior no pulmão que fica em plano inferior, com o pulmão afetado para baixo, não consegue aumentar, na mesma proporção de perfusão, levando a maior desproporção na relação ventilação e perfusão, e conseqüentemente, piora na troca gasosa (ZACK et alii 1974; KATZ \& BARASH 1977; REMOLINA et alii 1984).

No entanto, na situação de ventilação mecânica, a distribuição da ventilaçāo não ocorre da mesma forma que na respiração espontânea. Como a distribuição da ventilação, depende da complacência pulmonar, que é menor no pulmão que fica em plano inferior, o pulmão que está na posição superior, recebe maior ventilação que o de baixo (RHEDER et alii 1972).

Em relação à posição supina, neste trabalho, ela e a posição deitada sobre o lado não afetado, foram igualmente superiores à deitada sobre o lado afetado, o que discorda dos achados de DHAINAUT et alii (1980); IBANEZ et alii (1981) e REMOLINA et alii (1984), visto que encontraram que a posição deitada sobre o lado não afetado foi significativamente melhor que a supina.

Talvez, uma das razōes de termos obtidos melhora significativa da $\mathrm{PaO} 2$ na posição supina, seja o fato de que todos os pacientes com disfunção pulmonar unilateral, possuiam, entre outras, a base do pulmão afetada, em graus variáveis. Como a posição supina, provoca uma distribuição da ventilação e perfusão mais equitativas entre o ápice e a base pulmonar, talvez isto, tenha levado à uma melhor relação da ventilação e perfusão, nas áreas pulmonares não acometidas, com conseqüente melhora na $\mathrm{PaO} 2$.

Em relação à posição sentada, os valores da $\mathrm{PaO} 2$ foram menores, se comparados às posiçōes supina e deitado sobre o lado sadio, mas esta diferença não foi estatisticamente significativa. Não foi encontrado nenhum trabalho que tivesse estudado os efeitos da posição sentada sobre $\mathrm{PaO} 2$, em pacientes com doença pulmonar unilateral.

Para planejar a mudança de decúbito nos pacientes com disfunção pulmonar, o enfermeiro deve estar ciente da área pulmonar afetada e, optar pelas posiçōes que podem otimizar a relação ventilação e perfusão, e desta forma, melhorar a oxigenação ou prevenir hipoxemia. 
Assim, na disfunção pulmonar unilateral, pelos resultados obtidos, acreditase que o uso prioritário das posições deitado sobre o lado não afetado e supina, podem trazer benefícios para a oxigenação do paciente. Por outro lado, a posição deitada sobre o lado afetado, por ter apresentado os menores níveis de oxigenação e por ser potencialmente hipoxêmica, deve ser evitada.

$O$ período de tempo que o paciente pode permanecer num posição considerada favorável para a oxigenação, sem que os níveis da $\mathrm{PaO} 2$ se alterem, ainda não foi determinado.

Cumpre lembrar que não foi objetivo deste estudo, determinar a correlação entre as alteraçōes nas concentrações dos gases e o período de tempo que o paciente permanece numa determinada posição. Entretanto, pela importância deste conhecimento, acredita-se que devam ser elaborados trabalhos para esclarecer este aspecto.

Outrossim, sugere-se que os enfermeiros baseiem-se na avaliação clínica dos pacientes, na rotina do serviço em que trabalham e em sua experiência pessoal para a determinação do tempo em que o paciente deve ficar em cada posição, até que estudos sobre este assunto sejam feitos.

Em relação ao suporte ventilatório recebido pelos pacientes, percebe-se que este variou. Assim, 2 pacientes estavam em ventilação mecânica, e 3 não recebiam nenhum tipo de oxigenoterapia, estavam respirando espontaneamente, em ar ambiente (Tabela 4).

Para verificar se a $\mathrm{PaO} 2$ apresentada por, estes grupos extremos, em resposta à posição, estava interferindo no grupo geral, que apresenta $F(0,05 ; 3 ; 60)=$ 2,760 e $\mathrm{T} 2=2,863$ (Quadro 1), retirou-se estes pacientes e refez-se o tratamento estatístico. Assim, ao retirar-se os 3 pacientes que estavam respirando em ar ambiente, para o mesmo $F$ crítico, obtivemos $T 2=1,87972$. Ao se proceder de forma semelhante, agora retirando os 2 pacientes que estavam em ventilação mecânica, para o mesmo F crítico, obteve-se $T 2=3,101$. Estes dados sugerem que os pacientes que estavam respirando em ar ambiente, beneficiaram-se mais da posição, que os que estavam em ventilação mecânica, visto que ao serem retidados do grupo, o T2 diminuiu. Ao contrário, para os pacientes que estavam em ventilador mecânico, parece que o uso da posiçāo não interferiu, da mesma forma, pois ao serem retirados do grupo o $\mathrm{T} 2$ melhorou.

Talvez isto tenha ocorrido visto que os pacientes que estão em ventilação mecânica já apresentavam um suporte ventilatório importante, e o efeito da posição sobre a $\mathrm{PaO} 2$ diluiu-se. Outra hipótese para esta não interferência da posição, talvez seja devida ao fato de que na ventilação mecânica a distribuiçāo da ventilação está alterada. 
Cabe ressaltar, que devido ao pequeno número de ocorrências, estes dados são apenas sugestivos. No entanto, parecem suficientes para que novos estudos, que controlem o tipo de oxigenoterapia utilizada, sejam feitos.

Outro aspecto, que supōe-se ser importante para futuras pesquisas, é a verificação do efeito das posiçōes nas diferentes disfunçōes pulmonares ou pneumopatias, visto que a especificidade das alterações, talvez interfira na resposta.

\section{QUADRO 3 \\ COMPORTAMENTO DE PaCO2 EM RELAÇĀO ÀS POSIÇŌES \\ SÃO PAULO, 1987}

\begin{tabular}{lrrrr}
\hline & Sentada & Supina & $\begin{array}{l}\text { Lado não } \\
\text { afetado }\end{array}$ & Lado afetado \\
\hline Mediana & 36,50 & 38,00 & 36,80 & 38,00 \\
Média & 36,67 & 37,08 & 37,22 & 37,58 \\
Desvio padrão & 3,54 & 4,22 & 4,18 & 4,00 \\
Soma dos postos & 59,50 & 52,50 & 53,00 & 45,00 \\
No de vezes & 13 & 10 & 10 & 6 \\
2 MP* & 2 & 3 & 3 & 4 \\
No de vezes & 2 & & & \\
PP** & & & & \\
\hline
\end{tabular}

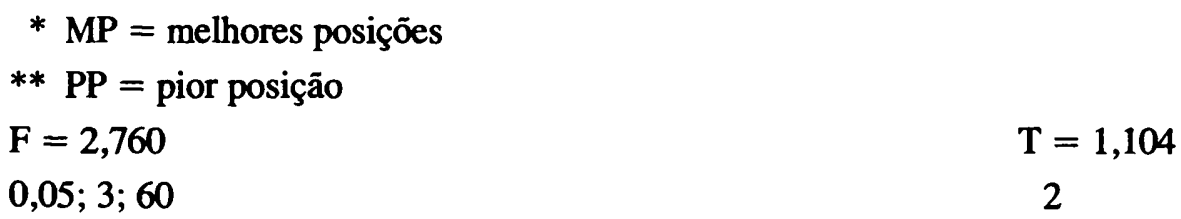

O teste de Friedman não apreendeu diferenças significativas, quanto à pressão de gás-carbônico entre as quatro posiçōes, ao nível de significância de 5\%.

Estes achados concordam com os de SEATON et alii 1979; IBANEZ et alii 1981 e REMOLINA et alii 1984, que também não encontraram variação significativa da $\mathrm{PaCO} 2$ nas diferentes posiçōes, nos pacientes por eles estudados.

É possível que haja relação entre a não variação da $\mathrm{PaCO} 2$ e a grande velocidade de difusão do gás-carbônico, que é maior que a do oxigênio 20 vezes. $O$ oxigênio no entanto, apresenta maiores limitaçōes à transferência, ligadas à perfusão (WEST 1977a). Como a mudança de posição interfere na distribuição da perfusão pulmonar, provavelmente afetará em maior grau a troca de $\mathrm{O} 2$. 


\section{CONCLUSŌES}

$\mathrm{O}$ estudo do efeito das posiçōes supina, sentada e laterais sobre a $\mathrm{PaO} 2$ e $\mathrm{PaCO} 2$, em 21 pacientes adultos, portadores de disfunção pulmonar unilateral, no pós-operatório recente de cirurgia cardíaca, permitiu as conclusões que se seguem:

- a $\mathrm{PaO} 2$ foi significantemente superior nas posições deitada sobre o lado não afetado e supina, ao nível de 5\%;

- a PaO2 foi significantemente inferior na posição deitada sobre o lado afetado, ao nível de 5\%;

- a PaCO2 não apresentou diferenças estatisticamente significantes nas 4 posiçōes, ao nível de 5\%.

Frente aos dados apresentados, recomenda-se que os enfermeiros incluam o uso de decúbitos específicos, no programa terapêutico dos pacientes com disfunçōes pulmonares, visto o benefício que esses podem proporcionar.

Em artigo subseqüente, apresentaremos os resultados relativos ao estudo do efeito da posição corporal sobre a oxigenação sangüínea, em portadores de disfunção pulmonar bilateral.

PIMENTA, C.A. de M. \& KOIZUMI, M.S. The effects of body positions on blood gas exchange in patients with unilateral lung disease - part I. Rev. Esc. Enf. USP, São Paulo, 23(3)205-221, dez. 1989.

The purpose of this study was to analyse the effects of sitting, supine and laterals positions on arterial oxygen tension $(\mathrm{PaO2})$ and arterial carbon dioxide tension (PaCO2), in 21 adults patients, carier of some pulmonary unilateral disfunction, in recent post-operative cardiac surgery. The diagnosis of the pulmonary disfunction was done through radiographic torax evaluation. The patients stayed during 15 minutes in each position and after this, the arterial blood gases were measured. Based on Friedman test and Multiple Comparation test $(\alpha=$ $0,05)$, it was found that the PaO2 was significantly higher when the patients were lying on the "healthy" side or in supine position; the $\mathrm{PaO} 2$ was significantly lower when the patients where lying on the "sick" side. No significant changes in $\mathrm{PaCO} 2$ were noted.

UNITERMS: Blood gas analysis. Patient position. Patient, posture changes.

\section{REFERÊNCIAS BIBLIOGRÁFICAS}

ARBORELIUS, M. et alii. Influence of unilateral hypoxia on blood flow through the lungs in man in lateral position. J. Appl. Physiol., Bethesda, 15(4): 595-7, July 1960.

BIDIWALA, K. et alii. Lateral decubitus position and pulmonary interstitial emphysema. J. Pediatr., Saint Louis, 105(5): 847-88, Nov. 1984.

CONOVER, W.J. Practical nomparamentric statistics. 2. ed. New York, John Wiley, 1980. 300p.

DEAN, E. Effect of body position on pulmonary function. Phys. Ther., Washington, 65(5): 613-18, May 1985.

Rev. Esc. Enf. USP, São Paulo, 23(3):205-221, dez. 1989 
DHAINAUT, J.F. et alii. Improved oxygenation in patients with extensive unilateral pneumonia using the lateral decubitus position. Thorax, London, 35(10): 792-3, Oct. 1980.

DICIONÁRIO Médico Blakiston. 2.ed. São Paulo, Andrei, 1982. 1169 p.

DOUGLAS, W.W. et alii. Improved oxygenation in patients with acute respiratory failure: the prone position. Am. Rev. Respir. Dis., New York, 115(4): 559-66, Apr. 1977.

FROESE, A.B. \& BRYAN, C. Effects of anesthesis and paralysis on diaphragmatic mechanics in man. Anyesthesiology, Philadelphia, 41(3): 242-55, Sept. 1974.

GOODNOUGH, S.K.C. The effects of oxygen and hyperinflation on arterial oxygen tension after endotracheal suctioning. Heart Lung, Saint Louis, 14(1): 11-7, Jan. 1985.

GROSMAIRE, E.K. Use of patient positioning to improve PaO2: a review. Heart Lung, Saint Louis, 12(6): 650-3, Nov. 1983.

IBANEZ, J. et alii. The effect of lateral positions on gas exchange in patients with unilateral lung disease during mechanical ventilation. Intensive Care Med., New York, 7(5): 231-5, Aug. 1981.

$\mathrm{KAHN}, \mathrm{A}$. et alii. Effects de la position ventrale sur les gas sanguins du nourrisson durant le sommeil. Arch. Fr. Pediatr., Paris, 42(6): 419-21, Juin/Juil. 1985.

KANEKO, K.J. et alii. Regional distribution of ventilation and perfusion as a function of body position. J. Appl. Physiol., Bethesda, 21(3): 767-77, May 1966.

KATZ, L.D. \& BARASH, P.G. Positional hypoxaemia following post traumatic pulmonary insuddiciency. Can. Anaesth. Soc. J., Toronto, 24(3): 346-52, May 1977.

MARTI, C. \& ULMER, W.T. Absence of effect of the body position on arterial blood gases. Respiration, Brasil, 43(1): 41-4, Jan./Feb. 1982.

NEAGLEY, R.S. \& ZWILLICH, W.C. The effect of positional changes on oxigenation in patients with pleural effusions. Chest, Park Ridge, 88(5): 714-17, Nov, 1985.

NG, L. \& McMCORMICK, K.A. Position changes and their physiological consequences. ANS, Germantown, 4(4): 13-25, July 1982.

NORTON, L.C. \& CONFORTI, C.G. The effects of body position on oxygenation. Heart Lung, Saint Louis, 14(1): 45-52, Jan. 1985.

PEIRANO, P. et alii. Effect of sleep position on transcutaneous oxygen tension in SIDS siblings. Early Hum. Dev., Amsterdam, 13(3): 303-12, June 1986.

RAY, J.F. et alii. Immobility, hypoxaemia and pulmonary arteriovenous shunting. Arch. Surg., Chicago, 109(4): 537-41, Oct. 1974.

RHEDER, K. et alii. The function of each lung of anesthetized and palyzed man during mechanical ventilation. Anesthesiology, Philadelphia, 37(1): 16-26, July 1972.

REMOLINA, C. et alii. Positional hypoxaemia in unilateral lung disease. N. Engl. J. Med., Boston, 304(9): 523-5, Feb, 1984.

SEATON, D. et alii. Effect of body position on gas exchange after thoracotomy. Thorax, London, 34(4): 518-22, Aug. 1979.

WAGNER, P.D. \& WEST, J.B. Changes in ventilation-perfusion relationships and gas exchange. In: MACKLEM, P. \& PERMUTT, S. The Iung in the transition between health and disease. New York, Marcel Dekker, 1979 . cap.9, p. 183-226.

WEST, J.B. Fisiologia respiratória moderna. São Paulo, Manole, 1977. p.26, 31 (a).

WEST, J.B. Ventilation perfusion relationships. Am. Rev. Respir. Dis., New York, 116(5): 919-43, Nov. 1977 (b).

WEST, J.B. \& DOLLERY, C.T. Distribution of blood flow and ventilation-perfusion ratio in the lung, measured with radioactive CO2. J. Appl. Physiol., Bethesda, 15(3): 405-10, 1960.

ZACK, B.M. et alii. The effect of lateral positions on gas exchange in pulmonary disease. Am. Rev. Respir. Dis., New York, 110(4): 49-55, Oct. 1974.

Recebido em 21/11/88 


\section{ANEXO I \\ Roteiro para coleta de dados}

I - Identificação

Nome .......... idade

sexo

reg

II - Caracterização da população

- Diagnóstico pulmonar

Grupo I - Doença unilateral

direita ( )

esquerda ( )

Grupo II - Doença bilateral

- Consciente

desperto ( )

inconsciente ()

- Cirurgia realizada

sonolento( )

- Drenos: sim ( ) não ( ) Localização

- Oxigenoterapia:

( )nenhum

( )ar ambiente

( )nebulização à distância $\mathrm{FiO} 2$

dias

( )máscara

( )ventilador

$\mathrm{FiO} 2$

Fluxo outros...

- Forma de obtenção das amostras de sangue: cateter ( ) punção ( )

III - Posição e respectiva $\mathrm{PaO} 2$ e $\mathrm{PaCO} 2$ (mmHg)

Posição

$\mathrm{PaO} 2$

$\mathrm{PaCO} 2$

sentada

supina

Lateral D

Lateral E

IV - Intercorrências 